\title{
Prácticas de labranza en el sur de Pozuelos (Puna de Jujuy, Argentina). Avances en el análisis de implementos agrícolas prehispánicos
}

\section{Carlos I. Angiorama*, Valeria L. Franco Salvi ${ }^{* * *}$ y Alexis A. Coronel $^{* * * *}$}

Recibido: 15 de febrero de 2018

Aceptado:

8 de octubre de 2018

\section{Palabras clave}

Implementos agrícolas Análisis lítico

Microrrestos

Puna de Jujuy

Moreta

Keywords

Agricultural tools

Lithic analysis

Micro-remains

Puna de Jujuy

Moreta

\footnotetext{
* Instituto de Arqueología y Museo, Facultad de Ciencias Naturales e Instituto Miguel Lillo, Universidad Nacional de Tucumán (UNT). San Martín 1545 (CP T4000CWE), San Miguel de Tucumán, Tucumán, Argentina. E-mail: carlosangiorama@gmail.com

** Instituto de Humanidades (IDH), Universidad Nacional de Córdoba (UNC) - CONICET. Haya de la Torre s/n, Pabellón Agustín Tosco, 1ำ piso, Ciudad Universitaria (CP X500oHUA), Córdoba, Argentina. E-mail: valefrancosalvi@unc.edu.ar *** Instituto Superior de Estudios Sociales (ISES), Facultad de Ciencias Naturales e Instituto Miguel Lillio, Universidad Nacional de Tucumán (UNT) - CONICET. San Lorenzo 429 (CP T40ooCWE), San Miguel de Tucumán, Tucumán, Argentina. E-mail: alenel12@hotmail.com
} 
1. Esta última línea de trabajo fue abordada por Babot sobre manos de moler, conanas y cuchillos/ raederas de módulo grandísimo (Babot, 2007, 2009; Babot, Escola y Hocsman, 2008).

\section{Introducción}

Nuestros trabajos en el sur de Pozuelos, iniciados en 2005, permitieron el hallazgo en superficie y en excavaciones estratigráficas de numerosos implementos agrícolas. Sobre ellos se han realizado estudios sistemáticos que permitieron aportes teórico-metodológicos valiosos acerca de diversos aspectos de estos artefactos (Ávalos, 2010, 2016a, 2016b). En este artículo presentamos un estudio integral sobre un nuevo conjunto de 11 implementos agrícolas, no incluidos en los trabajos anteriores, obtenidos en los últimos años mediante excavaciones estratigráficas propias en el sitio arqueológico conocido como Moreta. Este asentamiento se encuentra localizado en el sector sur de la cuenca de Pozuelos (Puna de Jujuy) y estuvo habitado al menos desde mediados del primer milenio de nuestra Era hasta tiempos coloniales tempranos (Angiorama, Pérez Pieroni y Becerra, 2018; Pérez Pieroni y Angiorama, en prensa). Presentamos una síntesis que integra el análisis de los artefactos con un estudio arqueobotánico de adherencias a sus filos y con las características de los contextos en los que fueron hallados, a fin de avanzar en nuestro conocimiento acerca del rol que jugaron estos objetos en la vida cotidiana en sur de Pozuelos.

Hasta el momento, en la Puna argentina se han realizado estudios sistemáticos de implementos de labranza desde diversas perspectivas teórico-metodológicas. Entre los diferentes abordajes se destacan aquellos efectuados desde la organización tecnológica, la biografía de objetos, los trabajos de experimentación, los estudios tecno-morfológicos y los traceológicos (Ávalos, 1998, 2010, 2016a, 2016b; Fabrón, 2012; Haber y Gastaldi, 2006; Pérez, 2004, 2006, 2008, 2015), quedando pendiente aún la realización de análisis arqueobotánicos de residuos adheridos a los filos de los artefactos ${ }^{1}$.

Un trabajo pionero sobre el tema fue el efectuado por Yacobaccio (1983), quien estudió una amplia muestra de instrumentos de labranza procedentes de diversos sitios del Noroeste Argentino, a saber: Campo Colorado (Salta), Rodeo Colorado (Salta), Iruya (Salta) y Moreta (Jujuy).

También se han llevado a cabo estudios sobre herramientas agrícolas procedentes de ciertos sectores de la Puna de Jujuy cercanos al sitio de Moreta referidos al uso, función e importancia de estos artefactos líticos en la vida cotidiana. Uno de los primeros trabajos en este sentido fue el efectuado por Suetta y Alfaro de Lanzone (1979) sobre materiales hallados en el Pukara de Rinconada, otro de los asentamientos localizados en el sur de la cuenca de Pozuelos. Los autores plantearon que estos implementos presentaban allí dos formas diferenciadas, esto es, formas “típicas" y "atípicas" (Suetta y Alfaro de Lanzone, 1979): las palas, destinadas a un uso primario, o sea roturar la tierra, y los azadones, destinados a un uso complementario, como el corte previo a la recolección de ciertos vegetales.

Estudios posteriores han comenzado a cuestionar dicha interpretación a partir del "análisis biográfico de instrumentos" (Ávalos, 2010) indagando acerca de los cambios morfológicos de las palas a través del mantenimiento y reciclaje. Ávalos (2010) incluyó en su investigación una muestra recuperada por nuestro equipo en diversos sitios pequeños del sur de Pozuelos, registrando una amplia variedad morfológica de instrumentos enteros y fragmentados confeccionados sobre andesita basáltica. Concluyó que la variabilidad morfológica, en lugar de representar dos grupos de artefactos con función complementaria (Suetta y Alfaro de Lanzone, 1979), conformaría una diversificación de los anchos y de los contornos de los instrumentos (Ávalos, 2010). Esto significa que "las formas atípicas no son productos finales del proceso de manufactura, diseñados para cumplir una función complementaria a las de las formas típicas, sino que los implementos que componen las formas atípicas son tipos derivados o transformados" (Ávalos, 2010, p. 1615).

Es de destacar que Ávalos (2016a) analizó en total más de quinientos artefactos líticos del Período de Desarrollos Regionales (900-1430 A.D.) e Inka (1430-1535 
A.D.) de un gran sector del área Circumpuneña. La magnitud de la muestra y la sistematicidad en su estudio le permitió plantear también que los productos adquiridos de las canteras pudieron variar, siendo enmangados dichos implementos, preformas y blanks y utilizados en muchos casos aunque también puestos de forma precedente a formatización de acuerdo a un morfotipo particular (Ávalos, 2010a).

Resulta preciso mencionar también los trabajos de Pérez (2003, 2004, 2006, 2007, 2010) sobre la producción y variabilidad de implementos agrícolas y sobre la experimentación con palas y/o azadas líticas de dos sectores de la Puna argentina: las cuencas de Antofagasta de la Sierra (Puna meridional, Catamarca) y Doncellas (Puna de Jujuy). La autora se centró en el proceso de transición de las sociedades agropastoriles tempranas, enfocándose en aspectos tales como la tecnología involucrada en la manufactura de los instrumentos, desde un enfoque técnico-morfológico y morfológico-funcional, con el aporte del análisis de huellas de uso. Pérez argumenta que habrían sido "instrumentos para trabajar la tierra, abarcando una amplia gama de tareas posibles de realizar, que incluyen tanto aquellas relacionadas con la agricultura como las tendientes a responder a las necesidades de un campamento base, como por ejemplo picar, cavar, levantar, barrer, zapar, entre otras" (Pérez, 2010, p. 17).

En general, los trabajos sobre el tema se han enfocado en la identificación de la funcionalidad de las herramientas (en el sentido de la clase de artefacto: azada, pala u otro implemento) mediante el estudio de huellas de uso y/o en discusiones centradas en aspectos morfológicos y técnicos derivados de la manufactura de este tipo de herramientas. Sin embargo, Ávalos $(2016 \mathrm{a}, 2016 \mathrm{~b})$ ha comenzado recientemente a deconstruir el análisis de las palas buscando conocer, a través de ellas, cómo eran las prácticas agrícolas antiguas, relacionando la clase de herramienta con los gestos técnicos empleados en su uso, buscando ir más allá de la afirmación que plantea un uso-función para cavado y manipulación del sedimento. En este sentido, sostenemos que esa visión nos permite indagar acerca del rol de estos instrumentos en la vida cotidiana y comprender un poco más las escalas sociales de trabajo agrario existentes en el período estudiado.

A diferencia del énfasis en la literatura arqueológica por el análisis morfológicofuncional y de huellas de uso de los implementos agrícolas, son muy pocos aún los estudios sobre residuos orgánicos adheridos a los artefactos (i.e. fitolitos, granos de almidón, diatomeas, etc.). En este artículo abordamos esos tres aspectos, trabajando sobre ciertos implementos agrícolas hallados en nuestras excavaciones en Moreta ${ }^{2}$. Este tipo de abordaje permite acercarnos a conocer con mayor precisión las funciones primarias para las cuales fueron diseñados estos artefactos, orientándonos también acerca de las actividades en las que los objetos efectivamente participaron y, en algunos casos, acerca de las variedades cultivadas por los habitantes de los sitios trabajados.

\section{El sitio arqueológico Moreta}

La cuenca de Pozuelos es parte del extremo meridional del altiplano andino. En su porción sur se encuentra delimitada por las sierras de Rinconada y Carahuasi (al Oeste), Cochinoca (al Este) y Quichagua (al Sur). Hacia el Norte, la depresión ingresa en territorio boliviano sin solución de continuidad. Las prospecciones realizadas en este sector de la puna jujeña nos permitieron identificar un asentamiento arqueológico que hemos interpretado como el poblado y tambo de Moreta (Angiorama et al., 2018), excavado parcialmente por Márquez Miranda en $1945^{3}$.

El sitio está compuesto por un Recinto Perimetral Compuesto (RPC) de planta cuadrangular, de $60 \mathrm{~m}$ de lado, edificado junto a una serie de terrazas que nivelan el terreno circundante (Figura 1). Del otro lado de un curso de agua temporario
2. Los residuos microscópicos constituyen elementos que se adhieren al artefacto, a diferencia de las huellas de uso, las cuales siempre son el resultado de una pérdida de material en el mismo acto del uso (Babot, 2004). El filo constituye la parte del instrumento de labranza que tuvo contacto directo con los materiales orgánicos procesados.
3. No existen publicaciones que brinden detalles de la investigación realizada por Márquez Miranda en el sitio y sus anotaciones de campo no han sido halladas aún. En consecuencia, no conocemos qué tipos de contextos fueron excavados ni las asociaciones entre las piezas recolectadas. 


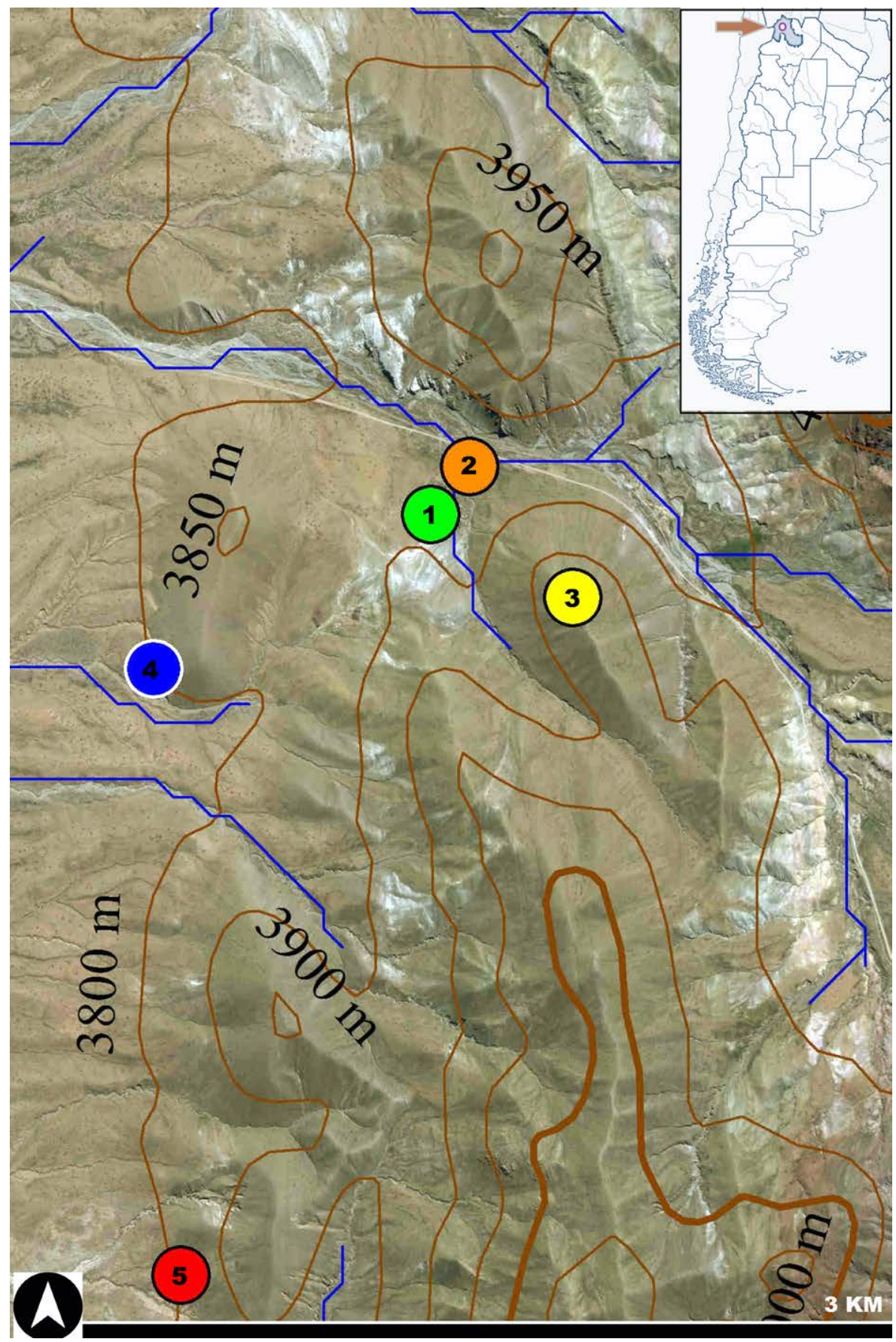

Figura 1. Sitio arqueológico Moreta. Referencias: 1) sector habitacional, 2) RPC, 3) sector defensivo, 4) sector agrícola más cercano al poblado, 5) sector agrícola más alejado del poblado.

se emplazan una serie de recintos que se aglutinan en un terreno de poco menos de 2 ha de superficie. Las estructuras habitacionales de ese sector del poblado presentan planta cuadrangular o rectangular y fueron construidas en torno a patios centrales cuadrangulares, configurando un patrón similar al registrado en otros lugares ocupados por poblaciones chicha en tiempos prehispánicos (Angiorama et al., 2018; Nielsen, Angiorama, Maryañski, Ávila y López, 2015). En este sector del asentamiento, a diferencia de lo que ocurre en el resto del sitio, la superficie se encuentra cubierta de fragmentos cerámicos, restos óseos de fauna y material lítico tallado y pulido (con abundantes fragmentos de instrumentos agrícolas). En 
el filo estrecho de la cima de una loma cercana que alcanza unos $120 \mathrm{~m}$ de altura respecto al poblado, se emplazan una serie de recintos habitacionales y muros de contención, conformando un sector de marcado carácter defensivo (Angiorama et al., 2018). En los márgenes del asentamiento se construyeronvarios corrales de grandes dimensiones, mientras que hacia el Sudoeste del RPC, en las lomadas más bajas de la Sierra de Cochinoca, se edificaron una serie de andenes y terrazas de cultivo que se extienden hacia el Sur, ocupando gran parte de cada una de las laderas orientadas hacia el bolsón de Pozuelos hasta la latitud de la localidad de Carahuasi, a unos 3,5 $\mathrm{km}$ de distancia. En algunos sectores, a los andenes y terrazas agrícolas se suman melgas en las porciones de menor pendiente cercanas a cursos de agua estacionales. La superficie ocupada por esta infraestructura agrícola supera las 100 ha y hasta el momento hemos hallado entre los campos de cultivo apenas unas siete estructuras que parecen haber sido recintos habitacionales o refugios agrícolas.

Los fechados radiocarbónicos realizados en los campos de cultivo más cercanos al poblado de Moreta indican que éstos datan del siglo XIV, lo cual es coherente con otros indicadores obtenidos en el sitio tanto en términos absolutos (Angiorama et al., 2018) como relativos (características arquitectónicas, recolecciones superficiales, etc.). Asimismo, hay una gran variabilidad de sectores de producción agrícola en el área que corresponden a distintos momentos cronológicos. Por ejemplo, al sur de los campos fechados, más alejados de Moreta, se registran sectores de producción agrícola de gran magnitud, sin parangón en el resto del sur de Pozuelos, que por su escala, diseño y manufactura es muy probable que daten de tiempos inkaicos. En este sentido, es de destacar que se emplazan junto a un tramo del Qhapaq Nan que atraviesa Pozuelos de Norte a Sur por el fondo de la cuenca, cercano a las últimas estribaciones occidentales de la Sierra de Cochinoca.

De acuerdo con nuestras observaciones, las excavaciones de Márquez Miranda se habrían concentrado en el sector del asentamiento donde se emplazan los conjuntos habitacionales de patrón chicha. Entre los numerosos hallazgos efectuados por ese investigador en 1945, se destacan por su cantidad los instrumentos líticos de labranza. Suman un total de 96, seis de los cuales fueron incluidos por Yacobaccio en su estudio pionero (Yacobaccio, 1983). Resulta interesante destacar que la mayoría de los implementos agrícolas recuperados en superficie por nosotros en el poblado de Moreta y sus alrededores, han sido hallados precisamente en el mismo sector de recintos conglomerados, estando prácticamente ausentes en las grandes áreas de cultivo cercanas. Una gran cantidad de los artefactos se encuentran fragmentados, mientras que aquellos enteros se han recuperado fundamentalmente en el interior de los recintos de habitación de patrón chicha excavados ${ }^{4}$.

\section{Contextos de hallazgo de los artefactos}

Del conjunto de más de un centenar de implementos agrícolas que hemos registrado durante los trabajos de campo en Moreta, nos referiremos en esta oportunidad sólo a los que fueron hallados en las excavaciones que hemos realizado en los últimos años. Se trata de un conjunto de 11 artefactos recuperados en sondeos estratigráficos efectuados en recintos domésticos que desde superficie se corresponden al patrón chicha. Es de destacar que, además, hemos sondeado uno de los recintos que componen el RPC, dos terrazas que se extienden entre el RPC y el sector chicha del poblado, un basurero localizado en el sector habitacional del sitio, un recinto adosado a un corral de gran tamaño y dos recintos asociados a la infraestructura agrícola más cercana al poblado. Tan solo en el basurero hemos hallado dos pequeños fragmentos de instrumentos de labranza. Esto es consistente con lo observado desde superficie, es decir, que este tipo de artefactos se concentra fundamentalmente en el sector residencial de patrón chicha del asentamiento.

Seis de los artefactos estudiados en esta ocasión proceden de la Estructura 1, excavada parcialmente. Se trata de un recinto de planta rectangular construido junto a otros
4. Albeck (2011) señala que también en Casabindo (Puna de Jujuy) hay una mayor frecuencia de implementos agrícolas en los recintos residenciales, en comparación con los dispersos en los campos de cultivo. 
. En todos los casos los fechados han sido calibrados a 1 sigma con el programa CALIB 7.0.4; Calibration data set: shcal13.14C (Hogg et al., 2013). similares en torno a un patio central cuadrangular delimitado por un muro bajo. El sondeo permitió identificar un piso de ocupación fechado en 1055-1270 cal d.C. ${ }^{5}$, correspondiente a la arquitectura chicha que se observaba desde superficie. Dos de los implementos agrícolas se hallaron sobre el piso de ocupación ( $\mathrm{N}^{\circ} 2$ y 3 ), mientras que tres se encontraron en el relleno posterior al abandono de la estructura ( No 9, 10 y 11). El restante ( $\left.\mathrm{N}^{\circ} 1\right)$ fue recuperado por debajo del piso fechado. Es preciso señalar que en este sondeo no se llegó al nivel estéril, permaneciendo aún inconclusa la excavación.

Otros tres implementos fueron recuperados en la Estructura 2, también excavada parcialmente. Al igual que la Estructura 1, se trata de un recinto edificado junto a otros en torno a un patio de características similares al descripto. A diferencia del caso anterior, aquí sí se completó la excavación hasta alcanzar el basamento estéril. Se detectaron tres pisos de ocupación superpuestos. El superior data de tiempos coloniales, a juzgar por una falange de fauna europea hallada sobre él. El piso medio, correspondiente a la arquitectura chicha observada en superficie, fue datado en 13131398 cal d.C. Para el piso inferior, correspondiente a otro tipo de arquitectura que en el sondeo solo se observó muy parcialmente, no poseemos dataciones aún. Uno de los instrumentos de labranza ( $\left.\mathrm{N}^{\circ} 7\right)$ fue hallado sobre el piso medio, mientras que los dos restantes $\left(\mathrm{N}^{\circ} 6\right.$ y 8$)$ fueron encontrados en el relleno depositado sobre el piso inferior.

Los dos artefactos restantes fueron recuperados en la Estructura 3, excavada parcialmente al igual que las otras dos. Se trata de un recinto construido junto a un patio cuadrangular, con características similares a los ya mencionados. En este caso también se alcanzó el nivel de basamento estéril. La excavación permitió identificar tres pisos de ocupación: los dos más modernos corresponden a la arquitectura observada en superficie, mientras que el más antiguo se asocia a un muro de características diferentes, con una orientación que no corresponde a la de las paredes superiores. Se obtuvieron dos dataciones radiocarbónicas para este sondeo. Una, correspondiente al piso superior, dio un resultado de 1017-1151 cal d.C. La otra, obtenida en un nivel que se extiende por debajo del piso medio, pero que no corresponde al piso inferior, dio un resultado de 970-1148 cal d.C. Uno de los artefactos de labranza (No 5) fue hallado sobre el piso de ocupación más moderno, mientras que el restante ( $\left.\mathrm{N}^{\circ} 4\right)$ fue encontrado en el relleno depositado luego del abandono del piso de ocupación medio.

\section{Primeros resultados}

\section{Análisis tecno-tipológicos y de huellas de uso}

Los artefactos fueron analizados siguiendo los lineamientos propuestos por Aschero (1975, 1983) para el análisis técnico-morfológico considerando: módulo longitud-anchura, materia prima, grupo tipológico, tamaño y espesor relativo, forma-base, características de las caras y referencias al contorno, etc. Por otra parte, Ávalos (1998, 2010, 2016a, 2016b) y Pérez (2007) nos guiaron en la identificación de la variabilidad de la muestra, de las huellas de manufactura (percusión, negativos de lascado, etc.) y uso (alisamiento, estrías, desprendimiento del material, etc.) y en la realización de inferencias funcionales. Los resultados obtenidos se combinaron luego con los estudios arqueobotánicos realizados en los filos de los instrumentos. El análisis de huellas fue realizado a nivel macroscópico con lupa binocular Schonfeld Optik Modelo STX-45 (entre 12 y 60x) para reconocer estrías y con lupa de mano para detectar alisamientos y brillos.

Con el fin de definir el tipo morfo-funcional de cada artefacto se determinó la relación ancho máximo y alto de la hoja y se procedió a describir las huellas de desgaste (Ávalos, 1998). Mangos y ataduras están completamente ausentes en nuestro registro material y son inferidos a partir de las características formales del diseño de las hojas y por las 
huellas de alisamiento y brillo en las caras de contacto de las mismas (Haber y Gastaldi, 2006). De manera preliminar, se caracterizaron las huellas de enmangue (Rots, Pirnay, Pirson, Baudoux y Vermeersch, 2001) a fin de conocer qué tipo de mango fue utilizado y por ende indagar en los gestos técnicos y las maneras en que fueron usadas estas herramientas. Asimismo, se contempló que ciertas morfologías pueden ser producto de actividades de mantenimiento/reparación artefactual aplicados a los tipos definidos (Ávalos, 2016b).

Ávalos (2016b, p. 2.609) sostiene que:

la configuración que adoptan las áreas desgastadas (en el cuerpo de la hoja y en el área del enmangue) definen patrones que pueden ser tratados como "diagnósticos" para interpretar el modo en que fueron enmangadas y usadas. De modo que diferenciar la cara ventral de la dorsal, el ángulo de ataque, el modo en que el filo entró en contacto con el sedimento (transversal u oblicuo) y la cara empleada para ser enmangada, son las vías por las cuales se puede reconstruir la estructura física de una herramienta así como los gestos técnicos asociados a su uso.

Este planteo resulta fundamental para conocer, más allá de la clase de herramienta, los "modos de uso" (Ávalos, 1998) de estos implementos.

Las herramientas analizadas se consideraron "enteras" cuando la porción faltante por la fractura de la hoja no supera el 10\% del total de la pieza (Ávalos, 2010). A las demás se las describió como "fracturadas". Los fragmentos se clasificaron en relación a la sección que se identifica (pedúnculo, segmento lateral o proximal de la hoja, etc.) y se observaron los retoques marcando los "primarios", "secundarios" o "post-manufactura" (Ávalos, 2010). Asimismo, se determinaron los tipos de fracturas, esto es: "directas" (radial, perversa, snap, etc.) e "indirectas" (end-shock, curvada o bending, etc.) (Weitzel y Colombo, 2006).

\section{Resultados}

\section{Materia prima}

Los 11 instrumentos fueron sometidos a un análisis macroscópico morfológicodescriptivo y se determinó que todos fueron confeccionados con andesita basáltica. Se trata de una materia prima cuya fuente no se encuentra a nivel local y hasta el momento desconocemos su procedencia. Presenta características óptimas, esto es, se trata de una materia prima granulada fina y compacta, lo que la hace adecuada para la formatización de las piezas y resistente para su uso en las tareas cotidianas.

\section{Manufactura}

Los instrumentos exhiben pátinas, marcas de uso -estrías, pulido, alisado, embotamiento del filo, esquirlamientos-y de enmangue, lo que de acuerdo con Ávalos (2010, p. 1.616) implica que "empezaron su vida útil como productos terminados". La ausencia en los recintos excavados y en las recolecciones superficiales de indicios del proceso de formatización inicial nos lleva a plantear como ya sostuvo Ávalos (1998) que "los instrumentos fueron confeccionados fuera del sitio (...) ya sea en canteras o talleres" (Ávalos, 1998, p. 293). Para el caso de la Puna de Jujuy, los productos obtenidos en las canteras serían preformas (Ávalos, 2016) de uno u otro de los tipos definidos para la región (Ávalos, 1998).

Los artefactos estudiados se han confeccionado mediante lascados marginales de formatización, tanto en el sector del pedúnculo como en los filos laterales. El sector 
del filo presenta un pulido intencional previo y la forma primaria del bisel sobre el filo de los instrumentos es simétrica en casi todos los casos. Los resultados que hemos obtenido acerca de las fracturas de los implementos son aún de carácter preliminar y hasta no obtener una colección experimental de referencia y efectuar estudios más específicos no es posible confirmar si las mismas se deben al uso, manufactura o procesos postdepositacionales. Basados en Pérez (2007, p. 81) iniciamos una descripción de fracturas principales y se registraron fracturas de tipo transversales tanto en la parte superior y media del cuerpo como así también en el cuerpo-pedúnculo y pedúnculo. Asimismo, detectamos fracturas longitudinales, diagonales y transversales del cuerpo aunque en menor proporción.

\section{Características de la forma, enmangamiento y modo de empleo}

En referencia a la morfología general de las piezas, predominan las trapezoidales y, en menor medida, las triangulares y/o rectangulares, con filos regularizados por abrasión uni o bifacial.

Se diferenciaron las áreas de desgaste de los implementos y se observó que cuatro de ellos fueron enmangados en la cara dorsal mientras que seis en la cara ventral (Ávalos, 2016b). Esto coincide con el análisis morfológico, es decir, los instrumentos que presentan un ancho de la hoja dos veces mayor a su largo (tipo A) fueron enmangados en la cara ventral, mientras que los que presentan un ancho tres veces mayor a su largo (tipo B) se enmangaron en la cara dorsal (sensu Ávalos, 2016b). Se infiere que aquellos que fueron enmangados en la cara ventral tuvieron un modo de empleo diferente a los que estuvieron amarrados desde la cara dorsal. En este último caso la mecánica fue clavar el artefacto - presión con un pie y apalancamiento- para levantar sedimento con un cabo recto, mientras que en el primer caso el método consistió en clavar y jalar la herramienta siendo el eje morfológico el que entra en forma perpendicular a la superficie.

De las tres piezas enteras analizadas, se observa que dos fueron utilizadas a modo de liukana o azada, esto es, con mango curvo y forma trapezoidal pedunculada, tipo A (Ávalos, 2016b). La pieza restante habría sido usada a modo de chakitaclla, con forma trapezoidal de tipo B (Ávalos, 2016b). Entre las piezas fragmentadas encontramos tanto algunas usadas a modo de chakitaclla como a manera de liukana o azada (Figura 2).

\section{Huellas de uso}

La mayoría de los implementos analizados tienen fracturas y presentan evidencias de filo activo con múltiples marcas (estrías, alisamiento, desprendimiento de material, etc.) (Weitzel y Colombo, 2006), las cuales fueron determinadas a partir de un análisis macroscópico morfológico descriptivo. El alto grado de fragmentación se debe, posiblemente, al uso de las mismas (sensu Yacobaccio, 1983). Las estrías detectadas se localizan en el cuerpo de la hoja y presentan una dirección perpendicular a la del filo, lo que indica una mecánica de movimiento unidireccional. La superficie altamente desgastada de los implementos y las fracturas de las herramientas líticas se explican también por las características del material sedimentario local, el cual presenta una textura arenosa con clastos que van aumentando su volumen y densidad con la profundidad del perfil. Los fragmentos de pala presentan fractura tabular, es decir, con sentido perpendicular al cual se aplicó la fuerza (Yacobaccio, 1983).

\section{Sustancias adheridas. Análisis arqueobotánico de los microrrestos obtenidos de los instrumentos de labranza}

Se realizó un análisis microscópico sobre el material sedimentario adherido a las superficies activas y pasivas de los instrumentos. Los artefactos no presentaban 


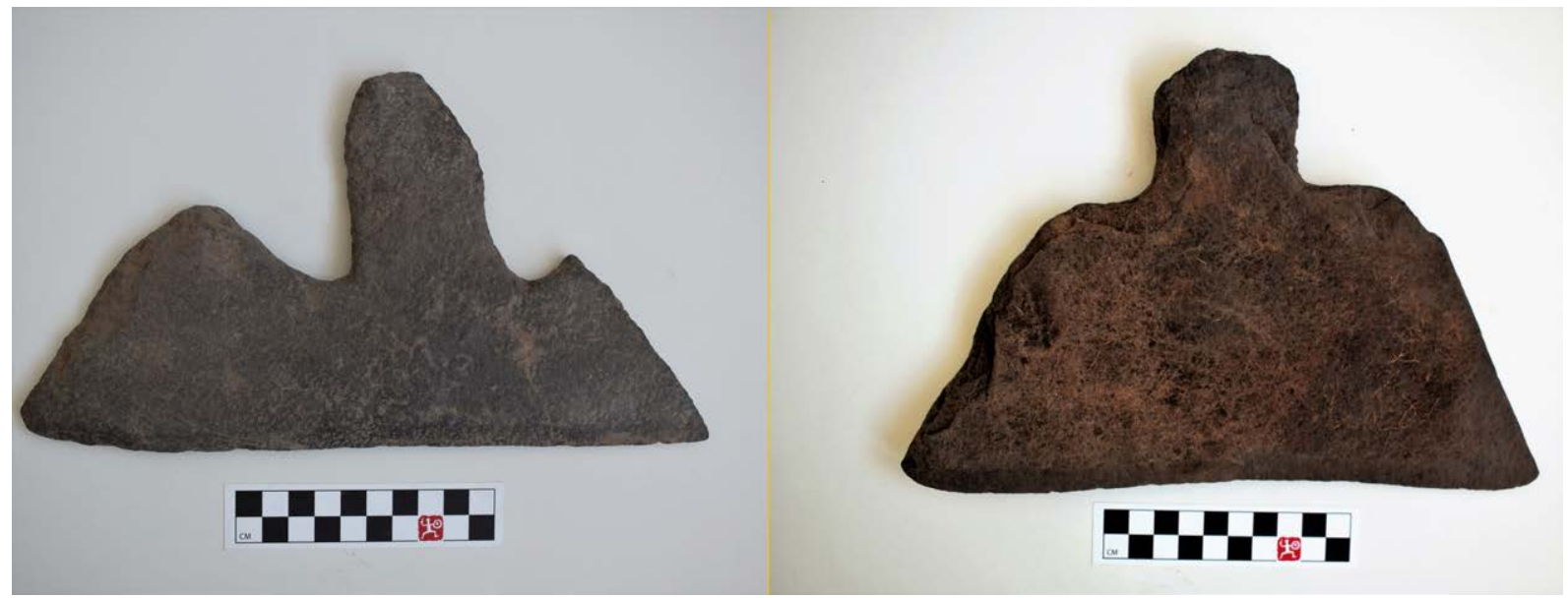

Figura 2. Ejemplos de los dos morfo-tipos diferenciados en la muestra. El instrumento de la derecha fue empleado con mango curvo a modo de liukana o azada y el de la izquierda a modo de chakitaclla (Avalos, 2016b).

adherencias visibles macroscópicamente, pero, sin embargo, fue posible obtener muestras de las "zonas de captura" (Escola, Hocsman y Babot, 2013), esto es, en negativos de lascado, fisuras y poros propios de la materia prima lítica (Babot et al., 2008). El procedimiento consistió en el raspado con aguja de acero sobre un área determinada en $\mathrm{cm}^{2}$ y en el montado directo de la muestra en un portaobjeto con aceite de inmersión, a fin de evitar posibles pérdidas de material (Babot, 2004). Los preparados fueron observados con microscopio óptico JPL1350 de polarización simple, a 400x de aumento.

Para la clasificación e identificación taxonómica de los cuerpos silíceos se procedió a su comparación con una colección de referencia y con material fotográfico y bibliográfico. Se empleó material de referencia del laboratorio del Instituto de Arqueología (IAMUNT) (Pigoni, 2009) realizado con vegetación de la localidad. Asimismo fueron consultados los trabajos de Twiss, Suess y Smith (1969) para gramíneas, Bozarth (1992) para dicotiledóneas y Ollendorf (1992) para ciperáceas. Para la identificación de especies cultivadas se utilizaron como guía los trabajos de Pearsall (2000), Piperno (1984, 2006), Rosen (1992) y Korstanje y Babot (2007).

Las evidencias arqueobotánicas microscópicas recuperadas de los artefactos estudiados consisten en silicofitolitos, granos de almidón y diatomeas. Los fitolitos (articulados y no articulados) constituyen el porcentaje más alto de la muestra y, en menor proporción, se detectaron granos de almidón y diatomeas.

En los filos de los implementos se diferenciaron silicofitolitos y granos de almidón con morfologías posibles de confrontar con las presentes en Chenopodiaceae (quínoa) y Amaranthus sp. (amaranto), hallándose diferentes partes anatómicas de esta planta: hoja y semilla. La asociación de fitolitos y almidones respalda la posibilidad de que esta especie haya sido cultivada en el lugar. Asimismo, se reconocieron formas afines a Zea mays (maíz) y Cucurbita sp. (zapallo), correspondientes al fruto de la planta (ver Figura 3 y Tablas 1,2 y 3 ).

También se identificaron fitolitos con morfotipos atribuibles a las familias de gramíneas silvestres de la región y de otras plantas domésticas. Se distingue un leve predominio de fitolitos Elongados (Prismatolita) y Chloridoideae (Saddle, Estrobilolita) por sobre los Panicoides (ver Tabla 4). Se observaron abundantes diatomeas continentales enteras y fragmentadas (v. g. Navicula sensu stricto) (Maidana, Seeligmann y Morales, 2011). 

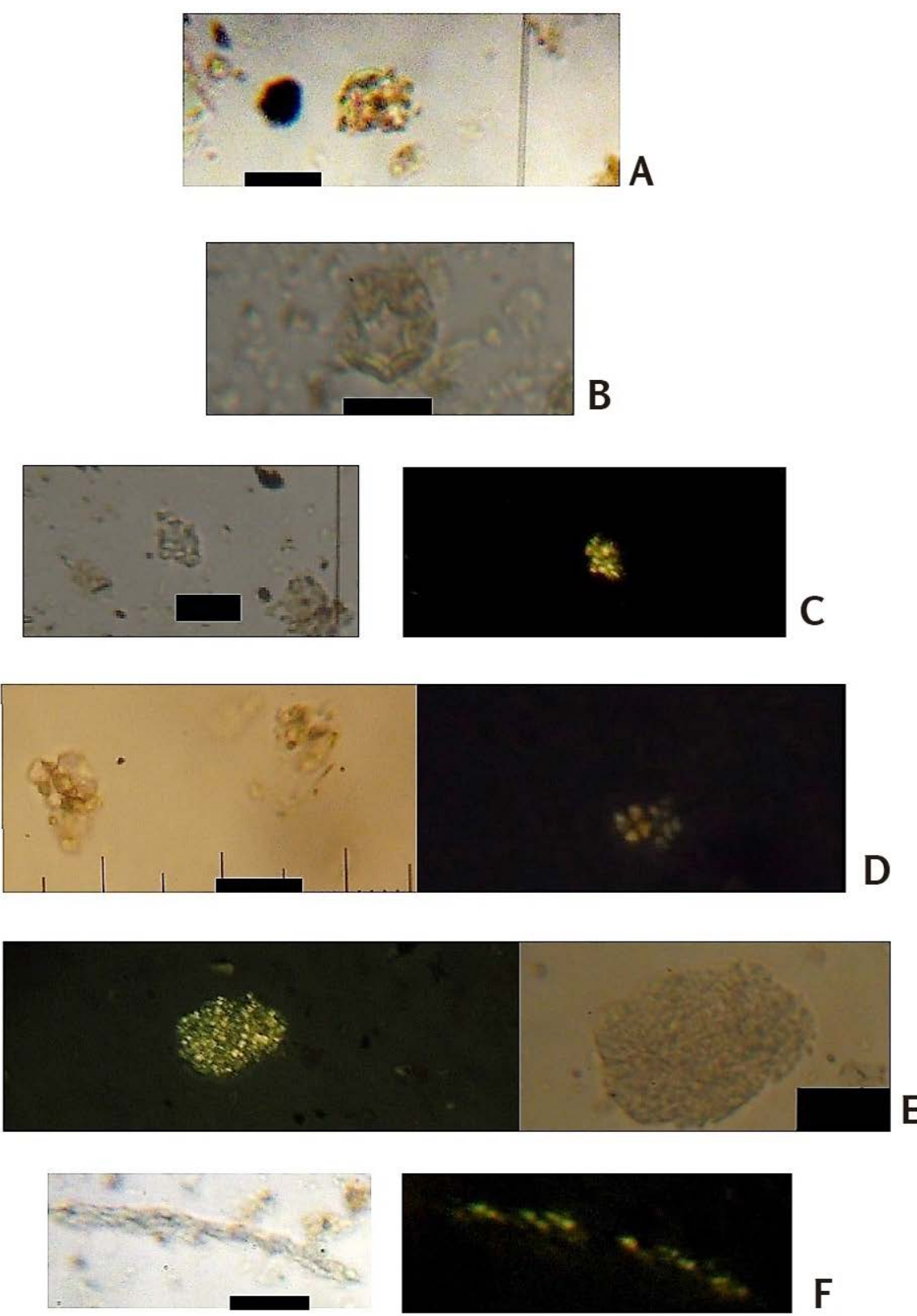

Figura 3. Fitolitos y gránulos de almidón reconocidos en los filos de los implementos agrícolas. Referencias: A) fitolito de Zea mays, B) fitolito de Cucurbita sp., C) gránulo de almidón de Amaranthus sp., D) gránulo de almidón de Amaranthus sp., E) y F) gránulos de almidón de Chenopodium quinoa.

\section{Discusión}

En nuestras excavaciones en el sitio arqueológico de Moreta hemos hallado dos clases de herramientas agrícolas que implican gestos de uso diferentes por los hábitos motrices que conlleva cada uno. Por un lado, un implemento empleado a modo de chakitaclla con cabo recto enmangado en la cara dorsal de la hoja y otro de mango curvo o en ángulo atado a la cara ventral a modo de liukana o azada (Ávalos, 2016b).

En términos generales, es posible sostener que estos instrumentos no se manufacturaron en el interior de las unidades residenciales donde fueron halladas. La ausencia en ellas, $y$ en el sitio en general, de indicadores del proceso de formatización inicial (desechos 


\begin{tabular}{|c|c|c|c|c|c|}
\hline \multirow[b]{2}{*}{ Artefacto } & \multirow[b]{2}{*}{ Lugar de hallazgo } & \multirow[b]{2}{*}{ Integridad } & \multicolumn{2}{|c|}{$\begin{array}{l}\text { Tipo morfofuncional } \\
\text { (Ávalos 2016b) }\end{array}$} & \multirow[b]{2}{*}{ Microfósiles } \\
\hline & & & $\begin{array}{c}\text { Implemento } \\
\text { empleado a modo } \\
\text { de chakitaclla }\end{array}$ & $\begin{array}{c}\text { Implemento } \\
\text { empleado a modo } \\
\text { de liukana/azada }\end{array}$ & \\
\hline 1 & $\begin{array}{l}\text { Estructura } 1 \\
\quad \text { UP } 105\end{array}$ & Entero & $x$ & - & $\begin{array}{l}\text { Chenopodiaceae (quínoa) } \\
\text { (fitolitos y granos de almidón) } \\
\text { Zea mays (maíz) (fitolito) }\end{array}$ \\
\hline 2 & $\begin{array}{l}\text { Estructura } 1 \\
\quad \text { UP } 104\end{array}$ & Entero & - & $x$ & - \\
\hline 3 & $\begin{array}{l}\text { Estructura } 1 \\
\quad \text { UP } 104\end{array}$ & Entero & $\mathrm{X}$ & - & $\begin{array}{l}\text { Cucurbita sp. (fitolito) } \\
\text { Amaranthus sp. (grano de } \\
\text { almidón) }\end{array}$ \\
\hline 4 & $\begin{array}{l}\text { Estructura } 3 \\
\quad \text { UP }_{103}\end{array}$ & Fragmento & - & $x$ & - \\
\hline 5 & $\begin{array}{l}\text { Estructura } 3 \\
\quad \text { UP }_{101}\end{array}$ & Fragmento & $\mathrm{X}$ & - & - \\
\hline 6 & $\begin{array}{l}\text { Estructura } 2 \\
\quad \text { UP } 104\end{array}$ & Fragmento & - & $x$ & $\begin{array}{l}\text { Amaranthus sp. (granos de } \\
\text { almidón) }\end{array}$ \\
\hline 7 & $\begin{array}{l}\text { Estructura } 2 \\
\text { UP }_{103}\end{array}$ & Fragmento & Indeterminado & Indeterminado & - \\
\hline 8 & $\begin{array}{l}\text { Estructura } 2 \\
\quad \text { UP } 104\end{array}$ & Fragmento & - & $x$ & $\begin{array}{l}\text { Chenopodium quinoa (granos de } \\
\text { almidón) }\end{array}$ \\
\hline 9 & $\begin{array}{l}\text { Estructura } 1 \\
\quad \text { UP }_{101}\end{array}$ & Fragmento & - & $\mathrm{x}$ & - \\
\hline 10 & $\begin{array}{l}\text { Estructura } 1 \\
\quad \text { UP }_{103}\end{array}$ & Fragmento & - & $x$ & $\begin{array}{l}\text { Chenopodiaceae (¿quínoa?, } \\
\text { fitolito) }\end{array}$ \\
\hline 11 & $\begin{array}{l}\text { Estructura } 1 \\
\quad \text { UP }_{102}\end{array}$ & Fragmento & $x$ & - & - \\
\hline
\end{tabular}

Tabla 1. Implementos agrícolas estudiados y microfósiles identificados en los filos.

\begin{tabular}{|c|c|c|c|}
\hline Artefacto & Identificación & Parte de la planta & Cantidad \\
\hline 1 & Chenopodiaceae & Hoja & 4 \\
& Zea mays & Fruto & 1 \\
\hline 3 & Cucurbita sp. & Fruto & 1 \\
\hline 10 & Chenopodiaceae & Semilla & 1 \\
\hline
\end{tabular}

Tabla 2. Fitolitos afines a plantas domésticas identificados en el filo de los instrumentos.

de talla, preformas o formas fallidas, etc.), coincide con lo señalado por Ávalos (1998) para otros sitios de la puna en los cuales plantea que su manufactura debió haberse llevado a cabo en las canteras.

El análisis arqueobotánico permitió identificar diferentes tipos de microfósiles en los implementos agrícolas. Es importante resaltar que ejemplares de ambos tipos de artefactos poseían en sus áreas activas restos de fitolitos y granos de almidón afines a quínoa y amaranto, aunque algunos de los implementos utilizados a modo de liukana o azada poseían en sus filos, además, restos de maíz y zapallo.

Los gestos técnicos vinculados al uso de herramientas con mango curvo son totalmente diferentes a los del uso de una herramienta con cabo recto. Por la evidencia compilada 


\begin{tabular}{|c|c|c|}
\hline Artefacto & Almidones & Identificación \\
\hline 1 & $\begin{array}{l}\text { Tres granos de almidón, ovoides y esféricos. Sus tamaños oscilan entre las } 18 \text { y } 25 \text { micras. No } \\
\text { se distingue hilum y lamella. }\end{array}$ & Chenopodium quinoa \\
\hline 3 & $\begin{array}{c}\text { Tres granos de almidón, esféricos y poliédricos de tamaños que oscilan entre las } 5 \text { y } 10 \\
\text { micras. Se encuentran agregados, lamella no visible. Cruz central con cuatro brazos visibles. } \\
\text { Corresponde a la semilla. }\end{array}$ & Amaranthus sp. \\
\hline \multirow{2}{*}{6} & $\begin{array}{l}\text { Cuatro agregados de almidones con forma esférica y poliédrica, facetados, con cruz céntrica } \\
\text { nítida con cuatro brazos visibles. }\end{array}$ & Amaranthus sp. \\
\hline & $\begin{array}{l}\text { Una estructura compuesta de forma incierta, hilo centrado como punto, cruz céntrica con } \\
\text { cuatro brazos visibles. Corresponde a la semilla. }\end{array}$ & Amaranthus sp. \\
\hline 8 & $\begin{array}{l}\text { Dos granos de almidón, ovoides y esféricos. Sus tamaños oscilan entre las } 18 \text { y } 25 \text { micras. No } \\
\text { se distingue hilum y lamella. }\end{array}$ & Chenopodium quinoa \\
\hline
\end{tabular}

Tabla 3. Granos de almidón identificados en el filo de los instrumentos.

\begin{tabular}{|c|c|c|c|c|c|c|c|c|c|c|}
\hline \multirow{2}{*}{ Microfósiles } & \multicolumn{10}{|c|}{ Artefacto } \\
\hline & 1 & 2 & 3 & 4 & 5 & 6 & 7 & 8 & 9 & 10 \\
\hline $\begin{array}{l}\text { Fitolitos Pooides } \\
\text { Rondels }\end{array}$ & 22 & - & - & 1 & - & 3 & - & - & - & 3 \\
\hline Fitolitos chloridoides Estrobilolita & 130 & 4 & 15 & 3 & 11 & 12 & 11 & 10 & - & 17 \\
\hline $\begin{array}{l}\text { Fitolitos chloridoides } \\
\text { Silla de montar (saddle) }\end{array}$ & 30 & - & 8 & 3 & 7 & 5 & 5 & 6 & - & 3 \\
\hline Fitolitos panicoides Bilobados & 23 & - & 10 & 2 & 5 & 2 & 3 & - & 1 & - \\
\hline Cruces (cross-chaped) & 1 & - & - & & 1 & - & 2 & - & - & - \\
\hline Polylobate & 22 & - & 3 & 1 & 5 & 1 & - & 3 & - & 1 \\
\hline Fitolitos Elongados (Prismatolita) & 39 & 12 & 65 & 6 & 43 & 26 & 26 & 20 & 6 & 20 \\
\hline Esféricos & 26 & 2 & 7 & 6 & 1 & 24 & 2 & 22 & 5 & 6 \\
\hline Polen & 3 & - & - & - & - & - & - & 1 & - & - \\
\hline Amorfos & - & 3 & 3 & - & 7 & - & 4 & - & - & - \\
\hline Esferas rugosas & 3 & - & - & - & - & 2 & 3 & - & - & - \\
\hline Fibra vegetal & - & 5 & - & - & - & - & - & - & - & - \\
\hline Cactaceae Opuntia sp. (Pasacana) & 1 & - & - & - & - & - & - & - & - & - \\
\hline Célula larga dendriforme & - & - & - & 2 & - & - & - & - & - & - \\
\hline Pelo silicificado & - & - & - & 2 & - & - & - & - & - & - \\
\hline Total & 298 & 26 & 111 & 26 & 80 & 75 & 56 & 62 & 12 & 27 \\
\hline
\end{tabular}

Tabla 4. Microfósiles correspondientes a gramíneas silvestres identificados en los filos activos de los instrumentos.

pensamos que la liukana o azada fue empleada principalmente para el "aporque" del maíz y del zapallo (arrimar tierra con nutrientes al pie de las plantas, mejorar el anclaje, eliminar malezas, etc.) y para la siembra de quínoa. Los registros etnográficos describen su efectividad al momento de colocar las semillas en cada hoyo (Vásquez, 1988).

Por otra parte, la chakitaqlla es un arado de pie o palo cavador (Vásquez, 1988) que cuenta con un timón y un soporte para el pie denominado "taquillpo" (Legoas Pena, 2015). Este instrumento se utiliza para roturar terrenos tanto planos como en pendiente (ver Figura 4). También se la emplea para la siembra, el aporque y la cosecha de tubérculos, y en menor escala, para cultivar granos. El gesto que implica su uso 

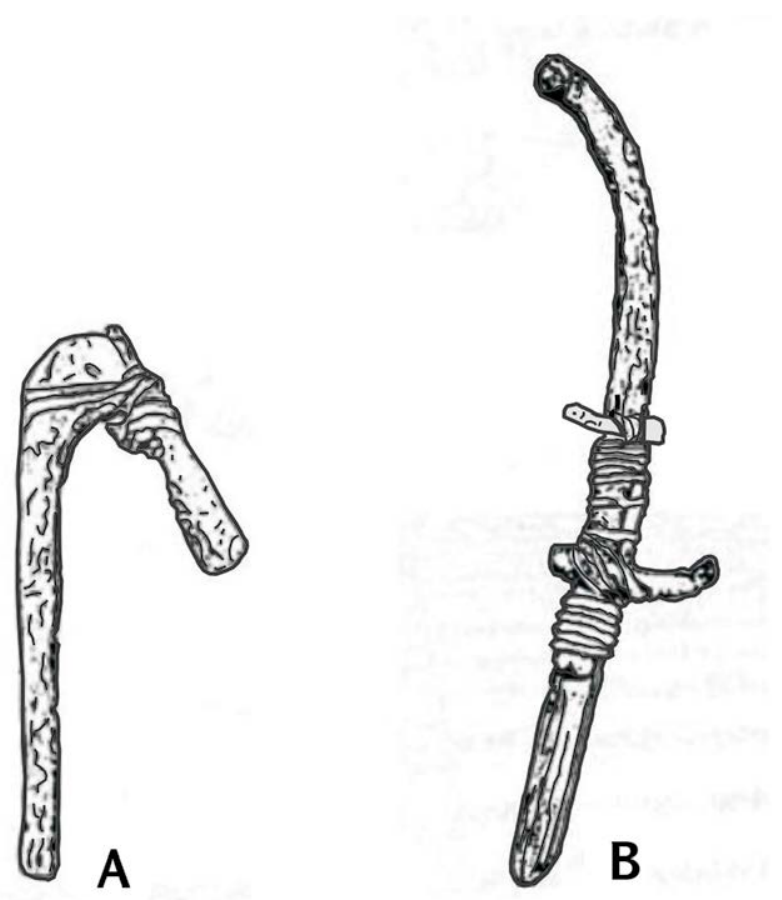

Figura 4. A) Likuana (tomada y modificada de la página web del Museo de Antropología de la Universidad de San Xavier de Chuquisaca, Bolivia. http:// www.ilam.org), B) Chakitaqlla (tomado y modificado de Vásquez 1988, p. 21).

es tomar el timón para levantar la herramienta y luego ejecutar un pequeño salto, impulsándose para pisar con el pie el "taquillpo", logrando la penetración del filo en el suelo, sacando champas que son volteadas por el rapeador/a. Este tipo de herramienta presentaba microfósiles afines a quínoa y amaranto.

Resulta llamativa la ausencia de restos de estos artefactos líticos en los campos de cultivo de gran escala. Como hemos dicho, durante nuestros trabajos de campo los fragmentos de implementos agrícolas recolectados en superficie fueron hallados casi exclusivamente en el sector residencial del sitio y, en menor medida, en las estructuras agrícolas más cercanas a él. Hasta el momento, solamente en los pisos de los recintos fue posible encontrar implementos enteros y esto podría relacionarse con prácticas de almacenamiento de las herramientas al finalizar la jornada laboral.

Es preciso señalar, sin embargo, que hasta ahora son escasos los sondeos estratigráficos que hemos realizado en los pocos recintos techables que se encuentran asociados directamente a las estructuras productivas. Futuras excavaciones a mayor escala permitirán evaluar la posibilidad de su uso como residencias temporales, como refugios para resguardarse durante las tareas agrícolas y/o como depósitos para el almacenamiento de los instrumentos de labranza.

En varios otros sitios del sur de la cuenca de Pozuelos contemporáneos a Moreta también hemos hallado implementos agrícolas, tanto en superficie como en excavación. Quisiéramos destacar de todos ellos, los primeros resultados obtenidos del estudio de los materiales recuperados en el sitio Tabladitas 1 (Angiorama y Becerra, 2014). En este pequeño asentamiento, en el que se observan unos pocos recintos residenciales de planta subcircular, asociados a estructuras agrícolas, depósitos construidos en oquedades y arte rupestre prehispánico tardío, los implementos agrícolas hallados en excavación (fechados en el siglo XIV de nuestra era) y los microfósiles asociados a ellos 
son casi idénticos a los de Moreta. La gran mayoría de los artefactos fueron hallados en la superficie del sitio se encontraban muy fragmentados, mientras que los recuperados en excavación estaban enteros. Al igual que en el caso de Moreta, las herramientas de Tabladitas 1 presentan forma trapezoidal pedunculada pero varían en el ancho y en el desgaste de las hojas - tipos A y B-. Algunos fueron utilizados con mango curvo a modo de liukana o azada y otros con mango recto a modo de chakitaclla (Ávalos, 2016b). En las muestras obtenidas de los filos de dichos instrumentos se observaron diversos microfósiles (fitolitos, almidones y diatomeas), destacándose la presencia de fitolitos afines a Cucurbita sp. y almidones con características posibles de confrontar con Amaranthus sp. (amaranto), Solanum tuberosum L. (papa) y Zea mays (maíz).

\section{Consideraciones finales}

El estudio de los implementos de labranza y de los microfósiles asociados a ellos nos permite conocer algo más acerca de ciertas prácticas de los habitantes de Moreta. En "lo cotidiano" (De Certeau, 1996) de cultivar es posible reconocer cómo las personas respondieron a diferentes acontecimientos locales y regionales. En este sentido, buscamos encontrar en las características de los implementos agrícolas aquellos indicios que nos cuenten acerca de las continuidades y rupturas que se manifestaron durante los períodos de Desarrollos Regionales e Inka con respecto a tiempos anteriores y posteriores. Durante los primeros siglos del segundo milenio de la Era, la población del sur de Pozuelos se distribuyó por el territorio en comunidades pequeñas, con una orientación pastoril y/o agrícola a baja escala (Angiorama, 2011). Sin embargo, estos diminutos caseríos convivieron con transformaciones políticas regionales que impactaron fuertemente en el área, como se puede observar claramente en el caso de Moreta, con la construcción de una gran infraestructura agrícola luego del arribo del inkario a la región. Si las escalas de trabajo se transformaron, resulta esperable que también lo hayan hecho los medios de producción. Sin embargo, en el caso de Moreta aparentemente se utilizaron los mismos tipos de herramientas agrícolas a lo largo de toda su ocupación. En este sentido, pese a la magnitud de los acontecimientos regionales, muchas de las prácticas cotidianas siguieron su curso sin denotarse cambios notables (Angiorama y Pérez Pieroni, 2013). Hasta el momento, en base a la evidencia arqueológica analizada, esto también se observa en las características de los artefactos agrícolas: no se registran modificaciones en sus morfologías, ni en su materia prima, ni en sus aspectos técnicos durante la larga secuencia de ocupación de Moreta, como sí ocurre en regiones cercanas como la Quebrada de Humahuaca, donde ingresaron nuevos tipos morfológicos durante el período Inka, provenientes de los Valles Orientales (Ávalos, 2016a).

Estos primeros avances nos alientan ahora a ampliar la muestra de estudio con materiales de otros contextos del sur de Pozuelos para su comparación. En este sentido, seguiremos indagando en distintas estrategias analíticas para caracterizar las secuencias de las actividades productivas que atraviesan los escenarios cotidianos en distintas escalas temporales (diaria, anual, vital) y las formas de interacción social involucradas en ellas, avanzando en el conocimiento de las estructuras - mecanismos generativos, lógicas- que fueron condición y resultado de estas prácticas habituales.

\section{Agradecimientos}

Las investigaciones fueron financiadas mediante subsidios PICT (FONCyT), PIP (CONICET) y PIUNT (Universidad Nacional de Tucumán). Agradecemos muy especialmente a cada uno de los habitantes del sur de Pozuelos por permitirnos trabajar en su territorio por más de una década y a todos los miembros del equipo que 
participaron en las tareas de campo realizadas en el área de estudio. Al Arq. Federico Bobillo y a la Lic. Rocío Molar por la lectura y sugerencias en aspectos de la especialidad en tecnología lítica y arqueobotánica. Damos las gracias muy especialmente al Lic. Julio Ávalos quien nos guió y acompañó en el estudio de los implementos líticos. Finalmente, a los/as evaluadores/as y editores/as que permitieron mediante sus observaciones mejorar el artículo. 


\section{Referencias citadas}

» Albeck, M. E. (2011). Estudios sobre Agricultura Prehispánica en Casabindo. En A. Korstanje y M. Quesada (Eds.), Arqueología de la Agricultura. Casos de Estudio en la Región Andina Argentina (pp. 12-47). San Miguel de Tucumán: Magna Ediciones.

" Angiorama, C. (2011). La ocupación del espacio en el sur de Pozuelos (Jujuy, Argentina) durante tiempos prehispánicos y coloniales. Estudios Sociales del NOA, 11, 125-142.

" Angiorama, C. I. y Becerra, M. F. (2014). “Como en ella jamás ha habido minas...” Minería y metalurgia en la Puna de Jujuy durante momentos prehispánicos tardíos. Relaciones de la Sociedad Argentina de Antropología, XXXIX, 313-332.

"Angiorama, C. I. y Pérez Pieroni, M. J. (2013). Primeros estudios sobre tecnología cerámica de contextos coloniales del sur de Pozuelos (Puna de Jujuy, Argentina). Revista de Arqueología Histórica Argentina y Latinoamericana, 6, 95-126.

"Angiorama, C. I., Pérez Pieroni, M. J y Becerra, M. F. (2018). Moreta, "pueblo de yndios chichas y tambo del ynga" (Puna de Jujuy, Argentina). Estudios Atacameños, 55, 163-181.

" Aschero, C. (1975). Ensayo para una clasificación morfológica de artefactos líticos aplicada a estudios tipológicos comparativos. Informe al CONICET. Manuscrito inédito.

" Aschero, C. (1983). Ensayo para una clasificación morfológica de artefactos líticos. Apéndices A y B. Apunte de la Cátedra de Ergología y Etnología (UBA), Buenos Aires. Manuscrito inédito.

"Ávalos, J. (1998). Modos de uso de implementos agrícolas de la quebrada de Humahuaca y Puna a través del análisis de huellas de desgaste. En M. B. Cremonte (Comp.) Los desarrollos locales y sus territorios. Arqueología del NOA y sur de Bolivia (pp. 285-303). San Salvador de Jujuy: Universidad Nacional de Jujuy.

" Ávalos, J. (2010). Vida útil y mutabilidad morfológica de los implementos de labranza agrícola: las "formas típicas" y "atípicas" del período tardío de la puna oriental (Pcia. de Jujuy, Argentina). En J. R. Bárcena y H. Chiavazza, Actas del XVII Congreso Nacional de Arqueología Argentina (Tomo 4) (pp. 1615-1620). Mendoza: Universidad Nacional de Cuyo.

» Ávalos, J. (2016a). Trayectorias de reducción de hojas líticas de herramientas agrícolas del período tardío e inka del área circumpuneña. Actas del XIX Congreso Nacional de Arqueología Argentina (pp.1436-1442). San Miguel de Tucumán: Facultad de Ciencias Naturales e Instituto Miguel Lillio.

»Ávalos, J. (2016b). Identificación de herramientas agrícolas y gestos técnicos asociados: una aproximación metodológica. Actas del XIX Congreso Nacional de Arqueología Argentina (pp. 2606-2612). San Miguel de Tucumán: Facultad de Ciencias Naturales e Instituto Miguel Lillio.

» Babot, M. P. (2004). Tecnología y Utilización de Artefactos de Molienda en el noroeste Prehispánico. (Tesis Doctoral inédita), Universidad Nacional de Tucumán, Argentina.

" Babot, M. P. (2007). Granos de almidón en contextos arqueológicos: posibilidades y perspectivas a partir de casos del Noroeste argentino. En M. B. Marconetto, M del P. Babot y N. Oliszewski (Eds.), Paleoetnobotánica del Cono Sur: estudios de casos y propuestas metodológicas (pp. 95-125). Córdoba: Ferreyra Editor. 
» Babot, M. P. (2009). Procesamiento de tubérculos y raíces por grupos agropastoriles del Noroeste argentino prehispánico: análisis de indicadores en residuos de molienda. En A. Capparelli, A. Chevalier y R. Piqué (Eds.), La alimentación en la América precolombina y colonial: una aproximación interdisciplinaria (pp. 67-81). Madrid: Consejo Superior de Investigaciones Científicas.

» Babot, M. P., Escola, P. y Hocsman, S. (2008). Microfósiles y atributos tecno-tipológicos: correlacionando raederas de módulo grandísimo con sus desechos de talla de mantenimiento en el Noroeste Argentino. En M. A. Korstanje y M. P. Babot (Eds.), Matices Interdisciplinarios en Estudios Fitolíticos y de Otros Microfósiles (pp. 187-200). Oxford: British Archaeological Reports.

» Bozarth, S. R. (1992). Classification of opal phytoliths formed in selected dicotyledons native to the Great Plains. En G. Rapp y S. C. Mulholland (Eds.), Phytolith Systematics (pp. 193-214). Nueva York: PlenumPress.

» De Certeau, M. (1996). La Invención de lo Cotidiano. 1 Artes de Hacer. México D. F.: Universidad Iberoamericana.

»Escola, P., Hocsman, S. y Babot, M. P. (2013). Entre las residencias y los campos de cultivo. Aportes de los cuchillos/raederas de módulo grandísimo a la cuestión de laboreo agrícola en Antofagasta de la Sierra (Puna de Catamarca) durante el primer milenio D.C. Relaciones de la Sociedad Argentina de Antropología, XXXVIII(1), 83-110.

»Fabron, G. (2012). Análisis de Tecnología Lítica: Palas y/o Azadas. Antumpa un caso de estudio (Pcia. de Jujuy). Anuario de Arqueología, 1, 205-218.

» Haber A. F, y Gastaldi, M. R. (2006). Vida con palas. Antípoda. Revista de Antropología y Arqueología, 2, 275-302.

»Hogg, A. G., Hua, Q., Blackwell, P. G., Niu, M., Buck, C. E., Guilderson, T. P., Heaton, T. J., Palmer, J. G., Reimer, P. J., Reimer, R. W., Turney, C. S. M. y Zimmerman, S. R. H. (2013). SHCal13 Southern Hemisphere Calibration, 0-50,000 Years cal BP. Radiocarbon, 55(4), 1889-1903.

» Korstanje, M. A. y Babot, M. P. (2007). Microfossils characterization from South Andean economic plants. En M. Madella y D. Zurro (Eds.), Plants, people and places: recent studies in phytolith analysis (pp. 41-72). Oxford: Oxbow.

»Legoas Pena, J. (2015). Ontographie du rapport à la terre à Chillimocco: Rationalité et rituel dans l'agriculture d'un village andin au Pérou. (Tesis Doctoral Inédita), Université Laval, Québec, Canadá.

» Maidana, N. I., Seeligmann, C. y Morales, M. (2011). El género Navicula sensu stricto (Bacillariophyceae) en humedales de altura de Jujuy, Argentina. Boletín de la Sociedad Argentina de Botánica, 46, 13-29.

» Nielsen, A., C. Angiorama, J. Maryañski, F. Ávila, y López, M. L. (2015). Paisajes Prehispánicos Tardíos en San Juan Mayo (Frontera Argentina - Bolivia). Arqueología, 21, $35-65$.

» Ollendorf, A. (1992). Toward a Classification scheme of sedge (Cyperaceae) phytoliths. En G. Rapp y S. C. Mulholland (Eds.), Phytolith Systematics (pp. 91-111). Nueva York: Plenum Press.

»Pearsall, D. M. (200o). Paleoethnobotany: A handbook of procedures. San Diego: Academic Press.

»Pérez, S. (2003). Experimentación y análisis de microdesgaste de palas y/o azadas líticas de Antofagasta de la Sierra (Catamarca). (Tesis de Licenciatura inédita), Universidad de Buenos Aires, Argentina. 
"Pérez, S. (2004). Experimentación de uso con palas y/o azadas líticas. Intersecciones en Antropología, 5, 105-117.

"Pérez, S. (2006). Arqueometría de palas y/o azadas líticas aplicada a estudios experimentales. El caso de Antofagasta de la Sierra (Catamarca). En A. Pifferetti y R. E. Bolmaro (Eds.), Actas del Primer Congreso Argentino de Arqueometría (pp. 230-240). Rosario: Universidad Nacional de Rosario.

"Pérez, S. (2007). Aportes metodológicos para el análisis de palas y/o azadas líticas. Mundo de Antes, 5, 73-89.

»Pérez, S. (2008). La organización de la tecnología lítica en el Noroeste Argentino. Aproximación a través de experimentación, análisis tecno- morfológico y de microdesgaste por uso de palas y/o azadas líticas. Comechingonia. Revista de Arqueología, 2(3), 186-222.

»Pérez, S. (2010). Variabilidad en la producción de palas y/o azadas líticas de la Puna argentina. Estudios Atacameños, 40, 5-22.

"Pérez, S. (2015). La organización de la tecnología lítica en el Noroeste argentino. Aproximación a través de experimentación, análisis tecno-morfológico y de microdesgaste por uso de palas y/o azadas líticas. Revista Arqueología, 21(2),307-309.

»Pérez Pieroni, M. J. y Angiorama, C. I. (en prensa). Evidencias arqueológicas de los siglos VII a XII AD en el asentamiento de Moreta (Puna de Jujuy, Argentina). Comechingonia. Revista de Arqueología.

» Pigoni, M. (2009). Análisis de microfósiles de muestras de sedimentos de los sitios Rio Herrana 5, Cerro Chiquito 2, Cienaga Rodeo 15 y Pan De Azucar 6. Informe al IAM-UNT. Manuscrito inédito.

»Piperno, D. (1984). A comparison and differentiation of phytoliths from maize and wild grasses: Use of morphological criteria. American Antiquity, 49, 361-383.

"Piperno, D. (2006). Phytoliths. A Comprehensive Guide for Archaeologists and Paleoecologists. Oxford: Alta Mira Press.

» Rosen, M. A. (1992). Preliminary identification of silica skeletons from Near Eastern archaeological sites: an anatomical aproach. En G. J. Rapp y S. C. Mulholland (Eds.), Phytoliths Systematics. Emerging Issues (pp. 199-147). Nueva York: Springer.

» Rots, V., Pirnay, L., Pirson, P., Baudoux, O. y Vermeersch, P. M. (2001).Experimental hafting traces. Identification and characteristics. Notae Praehistoricae, 21, 129-137.

»Suetta, J. M. y Alfaro de Lanzone, L. C. (1979). Excavaciones arqueológicas en el Pukara de Rinconada, Jujuy. Trabajo presentado en las Jornadas de Arqueología del NOA, Buenos Aires, Argentina.

"Twiss, P. C., Suess, E. y Smith, R. M. (1969). Morphological classification of grass phytoliths. Proceedings of the Soil Science Society of America, 33, 109-115.

"Vásquez, G. R. (1988). La agricultura tradicional en los Andes. Manejo de los suelos, sistemas de labranza y herramientas agrícolas. Notas para hipótesis de trabajo. Lima: Editorial Horizonte.

»Weitzel, C. y Colombo, C. (2006). ¿Qué hacemos con los fragmentos? Un experimento en fractura de artefactos líticos tallados. La Zaranda de Ideas. Revista de Jóvenes Investigadores en Arqueología, 2, 19-33.

» Yacobaccio, H. (1983). Estudio Funcional de azadas líticas del NOA. Arqueología Contemporánea, 1(1), 3-19. 